\title{
Relaying for 5G: A Novel Low-Error Relaying Protocol
}

\author{
Chunbo Luo ${ }^{\dagger}$, Gerard Parr ${ }^{\ddagger}$, Sally McClean ${ }^{\ddagger}$, Cathryn Peoples ${ }^{\ddagger}$, Xinheng Wang ${ }^{\dagger}$, James Nightingale ${ }^{\dagger}$, Qi Wang ${ }^{\dagger}$ \\ $\dagger$ School of Engineering and Computing, University of the West of Scotland, Paisley, PA1 2BE, UK. \\ †School of Computing and Information Engineering, University of Ulster, Coleraine, BT52 1SA, UK. \\ E-mail: \{chunbo.luo, xinheng.wang, james.nightingale, qi.wang\}@uws.ac.uk, \\ $\{c \cdot p e o p l e s$, gp.parr, si.mcclean\}@ulster.ac.uk
}

\begin{abstract}
Future 5G networks have stringent end-user requirements on data rate and error performance. In order to satisfy these requirements, innovative wireless networking technologies and models need be researched. One particular example is the two-way relaying channel, which can have as much as $100 \%$ higher theoretical data rate than current systems where transmissions are arranged in an orthogonal manner. However, benefits of this model cannot be achieved without the application of proper relaying protocols. This paper proposes a novel protocol that directly addresses the problems of existing protocols of twoway relaying models, e.g. analogy network coding and physical network coding, and has improved performance. By combining direct and differential demodulation-forward schemes based on wireless channel qualities and signal to noise ratio, a new hybrid protocol is created. Theoretical analysis and numerical experiments show that the proposed solution has lower error rate than the existing ones, and can thus be applied to support future 5G networks.
\end{abstract}

Index Terms-5G, relaying, two-way relaying, hybrid demodulation forward

\section{INTRODUCTION}

Industry and academia have much interest to research and develop future $5 \mathrm{G}$ technologies, which are planned to be standardized in 2020. One particular topic of 5G systems is the ambition to boost the capability of current cellular networks, which has become the bottleneck of mobile communications and struggle to satisfy the escalated demand from smart phones and large data applications including on-line gaming, social networking and content-share applications etc. In order to achieve this goal, numerous potential technologies need to be proposed and investigated, to name a few: relaying and cooperative communication, base station cooperation, cell size adaptation and multiple input multiple out etc. [1]. One particularly promising model is the Two-Way Relaying Channel (TWRC) [2], [3]. Fig. 1 shows an example of this model in one typical cell of cellular networks. Other examples include sensor networks and machine to machine communication scenarios [4], [5]. If TWRC is implemented properly through adequate protocols, its theoretical data rate can be $100 \%$ more than current systems where a pair of users have to be orthogonalized either in the time, frequency, code or space domain [6]-[8].

As shown in Fig. 1, a TWRC includes two source nodes (A and B) exchanging data through one intermediate relay (R). The two-way problem was first studied by Shannon [9]

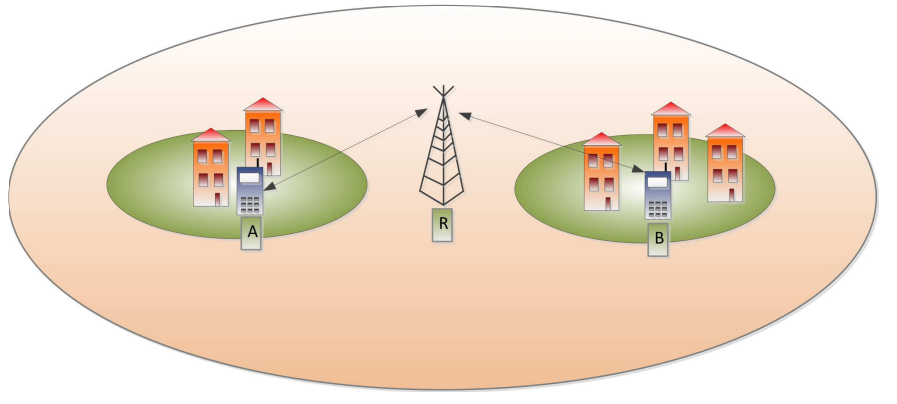

Fig. 1. Example of a two-way relaying channel within one cell.

and analyzed theoretically in terms of capacity by [10]. The introduction of relaying can significantly increase the performance of wireless networks if they are handled effectively by suitable protocols [11]. To this end, several relaying protocols, e.g. Amplify-Forward (AF) and Decode-Forward (DF) were proposed etc [12]. Both protocols can help achieve full diversity [13]. These fundamental protocols are often extended to more specific versions based on the applied network models.

Particularly for TWRC, several protocols have been proposed in terms of network coding in wireless domain: Analog Network Coding (ANC) [14], Physical-layer Network Coding (PNC) [15], Digital Network Coding (DNC) [16] and their equivalences. They can provide promising performance under ideal situations, for example, DNC based solutions have simple computation and signal processing on relays while PNC ones decode source messages before relaying and are less vulnerable towards error propagation. However, practical channel effects such as fading, Signal-to-Noise Ratio (SNR) variation and system implementation difficulties including imperfect synchronization may put obstacle to achieve the claimed advantages in real-life scenarios. For example, if channel gains of the two source-relay channels have large discrepancies, these schemes may incur large packet error rates and thus the spectrum efficiency would be greatly decreased by retransmitting large amounts of erroneous packets [15], [17]. This problem is particularly severe for the PNC protocol as it achieves the best performance when both channel gains are the same. It is therefore of theoretical interest and practical importance to design protocols/network coding schemes which can overcome the problems of wireless channels with dynamic 
fading and large SNR variations.

Inspired by the research on applying differential demodulation of received superposed signals to reduce noises [18][21], we propose a Hybrid DeModulate-Foward (HDMF) protocol for cellular networks to overcome the aforementioned problems that degrade the performance of existing TWRC protocols. The aim of this protocol is to achieve both high spectrum efficiency and low packet error rate. HDMF incorporates differential and direct DMF, and adapts the combining strategy based on the channel coefficients and SNR following the criterion of minimal packet-error. Theoretical analysis and numerical experiments confirm its performance over benchmark protocols. The proposed HDMF can be further extended to the hybrid-DF case, however, since this only increases the complexity (by adding a decoder) without bringing more understanding about the system, hybrid-DF is not covered in this paper.

This paper continues as follows: Section II describes the system model; Section III proposes the hybrid DMF protocol; Section V verifies its performance by numerical experiments; Section VI discusses the important issues; Section VII concludes the paper.

\section{SySTEM MODEL}

The system model considered in this work is the typical TWRC model, which has two source nodes (A and B) to exchange information through an intermediate relay (R) (Fig. 1). The channels are assumed to remain unchanged for at least one packet time slot and the channel coefficients are known at the corresponding destinations through, e.g. pilot based channel estimation. In designing the protocol, we assume noise to have a Gaussian distribution with zero mean and $N_{0} / 2$ variance.

At every time slot, source A and B simultaneously transmit data packets, e.g., $\mathbf{x}_{a}(n)$ and $\mathbf{x}_{b}(n)$ to relay R. Each packet has $M$ symbols, denoted as $\mathbf{x}_{a}(n)=\left[x_{a}^{1}(n), \cdots, x_{a}^{M}(n)\right]^{\mathrm{T}}$, $\mathbf{x}_{b}(n)=\left[x_{b}^{1}(n), \cdots, x_{b}^{M}(n)\right]^{\mathrm{T}} . \mathbf{R}$ receives the superposed version of the two packets from A and B as follows

$$
\mathbf{y}_{r}(n)=h_{A R}(n) \mathbf{x}_{a}(n)+h_{B R}(n) \mathbf{x}_{b}(n)+\mathbf{w}_{r}(n),
$$

where $\mathbf{y}_{r}(n)=\left[y_{r}^{1}(n), \cdots, y_{r}^{M}(n)\right]^{\mathrm{T}}, h_{A R}(n)$ and $h_{B R}(n)$ are the channel coefficients between A and R, B and R at the time slot $n . \mathbf{w}_{r}(n)$ is the noise vector at Relay. The $m$ th symbol in $\mathbf{y}_{r}(n)$ can be denoted as $y_{r}^{m}(n)$.

Messages received at Relay are processed and forwarded to the corresponding destinations by protocols, such as DMF, PNC and ANC. The forwarded signal $\mathbf{x}_{r}(n)$ is given by the following general case

$$
\mathbf{x}_{r}(n)=f\left(\mathbf{y}_{r}(n-1)\right),
$$

where $\mathbf{y}_{r}(n-1)$ is the received signal by Relay at the $(n-1)$ th time slot and $f(\cdot)$ is the relaying function which describes processes of the relaying protocol. For example, if using direct DMF, $f(\cdot)$ denotes the process of demodulating the received signal and then re-modulating it using the designated modulation scheme. In this case, data from the other user will not be detected. However, such case only happens if the channel quality deteriorates to a considerably low level as denoted by the likelihood ratio value. In order to ensure the dropped data can be retransmitted, current communication systems usually adopt Automatic Repeat reQuest (ARQ) schemes which are also assumed in this paper. The implementation details of ARQ are omitted here and can be found in [22]. If the weaker channel becomes strong enough, differential DMF will be automatically switched, where $f(\cdot)$ denotes the demodulation and remodulation of the data from both users. Even though sequential interference cancellation techniques can be used, it is not the focus here since the error rate of the weaker channel would be even higher than the stronger one.

The received signals at source B and A at the $n$th time slot are given by

$$
\begin{aligned}
& \mathbf{y}_{b}(n)=h_{R B}(n) \mathbf{x}_{r}(n)+\mathbf{w}_{b}(n), \\
& \mathbf{y}_{a}(n)=h_{R A}(n) \mathbf{x}_{r}(n)+\mathbf{w}_{a}(n),
\end{aligned}
$$

where $\mathbf{y}_{b}(n)=\left[y_{b}^{1}(n), \cdots, y_{b}^{M}(n)\right]^{\mathrm{T}}, h_{R B}(n)$ is the channel coefficient between $R$ and $B$ at the $n$th time slot, and $\mathbf{w}_{b}(n)$ is the noise vector at node B. $\mathbf{y}_{a}(n)$ and $h_{R A}(n)$ have similar definitions as those for source B.

\section{Hybrid Demodulation-Forward Protocol}

This section proposes the HDMF protocol. The general idea of HDMF is to have two DMF modes including direct DMF and differential DMF, and switch the two modes automatically based on a criterion obtained from the channel gains and SNRs. The protocol is implemented on the Relay and end nodes respectively, where the Relay uses it to construct and forward signals. After the reception of these signals, A and B apply the protocol to detect the source data. We use Quadrature Phase Shift Keying (QPSK) as an example to explain the mechanism of this protocol which can be easily extended to higher-order modulation scenarios.

To introduce the principles of this protocol: we assume that data packets from the two source nodes are of the same length. (The case of unequal length packets will be discussed in Section VI.) Each packet has $M$ symbols, and each symbol denotes $K$ bits if the modulation scheme has $K$-th order. For example, the source node packet $\mathbf{x}_{a}(n)$ has $M$ symbols and the $m$ th symbol $x_{a}^{m}(n)$ has $K$ bits which are denoted as $\left\{b_{a, 1}^{m}(n), \cdots, b_{a, K}^{m}(n)\right\}$. Similarly, $x_{b}^{m}(n)$ has $K$ bits and are denoted as $\left\{b_{b, 1}^{m}(n), \cdots, b_{b, K}^{m}(n)\right\}$. We assume the symbols are generated randomly with equal probabilities. Therefore, for Quadrature Phase Shift Key (QPSK) modulation, each symbol has two bits since $K=2$, and the four different constellation symbols of QPSK are generated randomly with the same probability: $\frac{1}{4}$.

The following subsections first introduce the two component parts of the HDMF protocol, and then present the decision criterion to choose these two components and the implementation details.

\section{A. Direct Demodulation-Forward}

The received signal at $\mathrm{R}$ is shown in (1). As two signals arrive simultaneously, we should try to demodulate the stronger signal directly in order to control the detection errors if one 
channel has significantly higher channel gain than the other and the noise is strong. Without loss of generality, we take the route from $\mathrm{A}$ to $\mathrm{R}$ and then from $\mathrm{R}$ to $\mathrm{B}$ as an example.

The maximum likelihood (ML) criterion is used to demodulate the $m$ th symbol within $\mathbf{x}_{a}(n)$,

$$
\hat{x}_{a}^{m}(n)=\arg \max _{x_{a}^{m}(n) \in \mathcal{M}}\left\{P\left(y_{r}^{m}(n) \mid x_{a}^{m}(n)\right\}, m=1, \ldots, M .\right.
$$

where $\mathcal{M}$ is the modulation symbol set. For QPSK, $\mathcal{M}=$ $\{1+i,-1+i,-1-i, 1-i\}$. Therefore, direct DMF has the forwarding signal as follows

$$
\mathbf{x}_{r}(n)=\hat{\mathbf{x}}_{a}(n)=\left\{\hat{x}_{a}^{1}(n), \ldots, \hat{x}_{a}^{M}(n)\right\}^{\mathrm{T}},
$$

which can replace the $f(\cdot)$ part of (2).

In order to describe the scheme concisely, the symbol index $m$ is omitted henceforward and $x_{a}$ always denotes the mth symbol within $\mathbf{x}_{a}$.

\section{B. Differential Demodulation-Forward}

This subsection introduces the differential DMF scheme for the two-way relaying model. Fundamentals of this scheme are introduced using QPSK as an example.

1) Differential DMF at Relay: In order to demonstrate the differential DMF algorithm, we firstly consider an instance where there is no noise and $h_{A R}(n)=h_{B R}(n)$. In this case, the two source signals of (1) arrive with the same power at Relay. The received superposed signal can be denoted as

$$
\mathbf{y}_{r}(n)=h_{A R}(n)\left(\mathbf{x}_{a}(n)+\mathbf{x}_{b}(n)\right) .
$$

For a given modulation scheme like QPSK, both the real and imaginary part of $y_{r}(n)$ only have two possible absolute values: $2\left|h_{A R}(n)\right|$ and 0 respectively. These two values correspond to the two input sets: $b_{a, k}(n)=b_{b, k}(n)$ and $b_{a, k}(n) \neq$ $b_{b, k}(n)$. It is easy for the relay to directly demodulate the received symbols at the first instance as both of them are the same and the power is enhanced. However, the latter case adds difficulty as it may be the superposition of two different bit sets: $b_{a, k}(n)=1, b_{b, k}(n)=0$ and $b_{a, k}(n)=0, b_{b, k}(n)=1$, $(k=1,2)$. However, since both the two sources know their transmitted signals, it is desirable for both A and B that Relay differentially demodulates and forwards signals to them.

The differential DMF at Relay, after converting data symbols to data bits, is given as

$$
\hat{b}_{r, k}(n)=\hat{b}_{a \oplus b, k}(n)=b_{a, k}(n) \oplus b_{b, k}(n), k=1,2,
$$

where $\oplus$ is the XOR (exclusive OR) operation. For example, if node $\mathrm{A}$ has a symbol $1+\mathrm{i}$ and node $\mathrm{B}$ has $-1+\mathrm{i}$, the corresponding bits of A's symbol are 1,1 and those of B's symbol are 0,1 . The resulting two bits after the above processing can be given as $\hat{b}_{r, 1}(n)=1 \oplus 0=1$ and $\hat{b}_{r, 2}(n)=1 \oplus 1=0$.

The above signal processing can be applied directly in the symbol domain if we can demodulate the differential between

$$
\begin{aligned}
& b_{a, k}(n) \text { and } b_{b, k}(n) \text { as follows } \\
& \quad b_{a \oplus b, k}(n)= \\
& \left\{\begin{aligned}
0, \operatorname{Re}\left\{y_{r}(n)\right\}=2 \operatorname{Re}\left\{h_{A R}(n)\right\} ; & 1, \operatorname{Re}\left\{y_{r}(n)\right\}=0 \\
0, \operatorname{Im}\left\{y_{r}(n)\right\}=2 \operatorname{Im}\left\{h_{A R}(n)\right\} ; & 1, \operatorname{Im}\left\{y_{r}(n)\right\}=0
\end{aligned}\right.
\end{aligned}
$$

where $\operatorname{Re}\{\cdot\}$ and $\operatorname{Im}\{\cdot\}$ denote the real and imaginary part of the input, respectively. The first line of (8) is for the first bit and the second line is for the second bit because one QPSK symbol denotes two bits.

The ideal case to employ differential DMF is when the two channel gains are the same, e.g. $\left|h_{A R}\right|=\left|h_{B R}\right|$, (where direct DMF has the worst performance) so that the majority of the received symbols will be far away from the decision lines. The worst case is when one of them is zero, e.g. $\left|h_{B R}\right|=0$. A small noise will push the signals from the correct side to the wrong side which are closely located around the decision lines, and thus generating a large error rate of $\frac{3}{8}$.

2) Detection of differential DMF symbols at $A$ and $B$ : The signal received by the two terminal nodes $\mathrm{A}$ and $\mathrm{B}$ are given by (3). We use node $\mathrm{A}$, for example: $\mathbf{y}_{a}(n)=$ $h_{R A}(n) \mathbf{x}_{r}(n)+\mathbf{w}_{a}(n)$ and $\mathbf{x}_{r}(n)=f\left(y_{r}(n-1)\right.$ ) (given by (2)). At the $n$th time slot, if the differential DMF protocol is applied to generate $\mathbf{x}_{r}(n)$, we obtain $\hat{b}_{r, k}(n)=\hat{b}_{a \oplus b, k}(n-1)$ for the $k$ th bit of the $m$ th symbol of $\hat{\mathbf{x}}_{r}(n)$. These received symbols should be demodulated first and then detected by the differential DMF protocol.

Similar to ML detection used in direct DMF, the differential DMF symbols received at the destination are firstly detected using (4). After the ML detection, we have the estimated symbol $\hat{\mathbf{x}}_{r}(n)$. The equation of this step is similar to (4) and is neglected here.

The second step is to decode the original data bits of the source symbols $x_{a}(n-1)$ and $x_{b}(n-1)$ through an XOR operation as follows

$$
\begin{aligned}
& \hat{b}_{a, k}(n)=b_{b, k}(n-1) \oplus \hat{b}_{r, k}(n), k=1,2, \\
& \hat{b}_{b, k}(n)=b_{a, k}(n-1) \oplus \hat{b}_{r, k}(n), k=1,2,
\end{aligned}
$$

where $\hat{b}_{r, k}(n)$ is the $k$ th data bit of the estimated symbol $\hat{x}_{r}(n)$. The other parameters of the equations are $b_{a, k}(n-1)$ and $b_{b, k}(n-1)$, which are the source data bits transmitted by the corresponding nodes, respectively, at one time slot previously. Such data should be stored in source nodes for later use. We can then obtain the desired data through the XOR operation in (9).

Comparing the two DMF schemes, we can find that differential DMF and direct DMF form a complementary relationship: one excels when the other has poor performance. It is easy to know which one should be used for the extreme cases discussed before. However, for a practical fading TWRC model whose channels are fading, the scenario is usually between these extreme situations. Therefore, it is important to find the decision criterion for the appropriate application of HDMF.

\section{Implement HDMF at Relay}


As discussed above, direct and differential DMFs are more suitable for different channel settings of the TWRC model. A key problem is to choose the right DMF schemes. We use the Log-Likelihood Ratio (LLR) tool to build such criterion.

The LLR value of each bit of the symbols under direct DMF is calculated based on channel conditions and SNR of the received signals, as follows,

$$
\begin{aligned}
& \mathcal{L}_{\text {Dir } A}(n, k)=\log \frac{P\left(y_{r}(n) \mid b_{a, k}(n)=1\right)}{P\left(y_{r}(n) \mid b_{a, k}(n)=0\right)}, \\
& \mathcal{L}_{\text {Dir } B}(n, k)=\log \frac{P\left(y_{r}(n) \mid b_{b, k}(n)=1\right)}{P\left(y_{r}(n) \mid b_{b, k}(n)=0\right)},
\end{aligned}
$$

where $\mathcal{L}_{\operatorname{Dir} A}(n, k)$ is the LLR of the $k$ th bit of the $m$ th symbol from $\mathrm{A}$ at the current time slot and $\mathcal{L}_{\operatorname{Dir} B}(n, k)$ is that obtained from $\mathrm{B}$, where the index $m$ is omitted in the equations for simplicity. Similarly, the LLR value under differential DMF can be obtained as follows,

$$
\mathcal{L}_{D i f}(n, k)=\log \frac{P\left(y_{r}(n) \mid b_{a, k}(n) \neq b_{b, k}(n)\right)}{P\left(y_{r}(n) \mid b_{a, k}(n)=b_{b, k}(n)\right)},
$$

where $\mathcal{L}_{D i f}(n, k)$ is the LLR of the $k$ th bit under the protocol of differential DMF given $x_{a}(n)$ and $x_{b}(n)$.

It is easy to know that the sign of an LLR value under the ML criterion denotes the detected bit result and its absolute value denotes the degree of confidence [23]. For a packet containing several symbols, we can try to find the symbol with the minimum confidence, which would most likely cause high error rate.

The LLR values of all symbols (where each symbol has $K$ bits) in the received packet at Relay, e.g., $y_{r}(n),(M$ total symbols) under direct DMF are calculated as follows,

$$
\begin{gathered}
\mathcal{L}_{\text {DirA }}(n)=\sum_{k=1}^{K}\left|\mathcal{L}_{\text {Dir A }}(n, k)\right|, \\
\mathcal{L}_{\text {DirB }}(n)=\sum_{k=1}^{K}\left|\mathcal{L}_{\text {DirB }}(n, k)\right| .
\end{gathered}
$$

Similarly, the LLR values of differential DMF are calculated as

$$
\mathcal{L}_{\text {Dif }}(n)=\sum_{k=1}^{K}\left|\mathcal{L}_{\text {Dif }}(n, k)\right| .
$$

A typical digital communication system usually uses packets to transmit data. The decision rule for a packet to be processed through direct or differential DMF is given as follows

$$
\begin{gathered}
\min \left\{\left|\mathcal{L}_{\text {Dir }}^{m}(n)\right|, m=1, \ldots, M\right\}>\min \left\{\left|\mathcal{L}_{\text {Dif }}^{m}(n)\right|,\right. \\
m=1, \ldots, M\}, \quad \text { Direct DMF, } \\
\min \left\{\left|\mathcal{L}_{D i r}^{m}(n)\right|, m=1, \ldots, M\right\} \leq \min \left\{\left|\mathcal{L}_{D i f}^{m}(n)\right|,\right. \\
m=1, \ldots, M\}, \quad \text { Differential DMF, } \\
\left|\mathcal{L}_{\text {Dir }}^{m}(n)\right|=\max \left\{\left|\mathcal{L}_{\text {Dir } A}^{m}(n)\right|,\left|\mathcal{L}_{D i r B}^{m}(n)\right|\right\} .
\end{gathered}
$$

We select the smallest LLR value from one packet transmitted from the stronger channel under direct DMF and compare it with the smallest LLR under differential DMF. By using this criterion, the DMF scheme with a greater minimum value will be selected to forward data.

\section{HDMF Detection at End Nodes}

The Relay with HDMF protocol can generate and forward two different kinds of messages by using the direct or differential DMF. For the end nodes (A and B), it is essential to be able to detect which kind of messages are transmitted from Relay. A possible solution is to add one bit in the packet header at the Relay, which indicates the DMF scheme used. However, such method increases implementation complexity and changes the frame structure, which is less desirable for practical wireless systems. In this section, we propose a blind detection algorithm with low complexity.

At the end nodes, e.g., source $B$, the received packet $\mathbf{y}_{b}(n)$ is first demodulated and mapped to data bits. A Cyclic Redundancy Check (CRC) is then performed on these data bits. If it is correct, this message is processed by direct DMF at Relay. If it is not correct, the second detection attempt using differential DMF (See Section III-B2) will be carried out. The decoded data bits, e.g., $\hat{b}_{a, k}(n)$, are checked by the CRC. If it is correct, differential DMF is used in the relay. Otherwise, the packet has some error symbols and should be discarded.

Finally, we analyze the above detection algorithm to see whether it can function properly in real life conditions. For a packet with a large enough number of symbols, e.g., $\mathrm{M}=128$, it contains 256 bits under QPSK modulation. (Higher order modulation has even more data bits.) We assume the data bits are randomly generated with equal probabilities for 0 and 1 . A bit level XOR operation with another randomly generated data packet would change approximately $50 \%$ of the data bits. Therefore, for a packet with 256 bits, approximately 128 bits would be changed after the XOR operation. This would identify the difference between direct DMF and differential DMF schemes and the probability of making a mistake is quite low. (If we consider a simple system without source coding and channel coding, the error rate is approximately $1 / 2^{128}$. With coding, the error rate can be more than this value because of coding dependency.)

\section{THEORETICAL ANALYSiS}

This section analyzes the proposed scheme and try to establish the mathematical expression for the end-to-end instantaneous Symbol Error Rate (SER). It is easy to know that errors usually come from two phases: the multiple access phase and the broadcast phase. In order to simplify the analysis, we take Binary Phase-Shift Keying (BPSK) as an example. The error rate at relay is denoted by $P_{r}$ and the error rates at the destination node $\mathrm{A}$ and $\mathrm{B}$ are denoted by $P_{r a}$ and $P_{r b}$ respectively. The end-to-end SER from A to $\mathrm{B}$ can be expressed as follows

$$
P_{a b}=1-\left(1-P_{r}\right)\left(1-P_{r b}\right)-P_{r} P_{r b}
$$

Similarly $P_{b a}$ can be obtained. The instantaneous SER can be expressed as

$$
\begin{aligned}
P_{H D M F} & =\frac{1}{2}\left(P_{a b}+P_{b a}\right) \\
& =P_{r}+\left(0.5-P_{r}\right)\left(P_{r a}+P_{r b}\right)
\end{aligned}
$$


The average SER of HDMF can be obtained as

$$
\begin{aligned}
\mathrm{E}\left\{P_{H D M F}\right\}= & \int_{\alpha} \int_{\beta} \int_{\gamma} \int_{\zeta} P_{H D M F} \\
& f(\alpha) f(\beta) f(\gamma) f(\zeta) \mathrm{d} \alpha \mathrm{d} \beta \mathrm{d} \gamma \mathrm{d} \zeta
\end{aligned}
$$

where $\alpha=\left|h_{A R}\right|, \beta=\left|h_{B R}\right|, \gamma=\left|h_{R A}\right|$ and $\zeta=\left|h_{R B}\right|$, which follow Rayleigh distributions with the probability density function as $f(x)=\frac{x}{\delta^{2}} e^{-x^{2} /\left(2 \delta^{2}\right)}$, where $\delta^{2}$ is the Rayleigh distribution parameter. We can assume these channel variables are independent and identically distributed (i.i.d.), so that the above equation is then simplified to

$$
\mathrm{E}\left\{P_{H D M F}\right\}=\mathrm{E}\left\{P_{r}\right\}+\left(0.5-\mathrm{E}\left\{P_{r}\right\}\right)\left(\mathrm{E}\left\{P_{r a}\right\}+\mathrm{E}\left\{P_{r b}\right\}\right),
$$

where $\mathrm{E}\left\{P_{r a}\right\}, \mathrm{E}\left\{P_{r b}\right\}$ and $\mathrm{E}\left\{P_{r}\right\}$ are the average SER of the Relay-A channel, Relay-B channel and sources-relay channels, respectively.

It is easy to obtain the average error rate for the relay to source channels as follows,

$$
\begin{aligned}
\mathrm{E}\left\{P_{r a}\right\} & =\int_{\gamma} P_{r a} f(\gamma) \mathrm{d} \gamma \\
& =\int_{0}^{\infty} Q\left(\sqrt{\frac{\gamma^{2} \epsilon_{r a}}{\sigma_{r a}^{2}}}\right) f(\gamma) \mathrm{d} \gamma \\
& =\frac{1}{2}\left(1-\sqrt{\frac{\rho_{r a}}{\rho_{r a}+1}}\right)
\end{aligned}
$$

where $\rho_{r a}=\epsilon_{r a} / \sigma_{r a}^{2}$ denotes the signal to noise ratio (SNR) and $Q\{x\}=\frac{1}{\sqrt{2 \pi}} \int_{x}^{\infty} e^{-t^{2} / 2} \mathrm{~d} t$. Similarly

$$
\mathrm{E}\left\{P_{r b}\right\}=\frac{1}{2}\left(1-\sqrt{\frac{\rho_{r b}}{\rho_{r b}+1}}\right) .
$$

The average SER of the sources-relay channels composes of two components from the direct demodulation (A-Relay and B-Relay) and differential demodulation (A\&B-Relay),

$$
\begin{aligned}
\mathrm{E}\left\{P_{r}\right\}= & \int_{\alpha} \int_{\beta}\left(P_{a b r} p_{a b r}+P_{a r} p_{a r}+P_{b r} p_{b r}\right) \\
& \cdot f(\alpha) f(\beta) \mathrm{d} \alpha \mathrm{d} \beta,
\end{aligned}
$$

where $P_{a r}$ and $P_{b r}$ are the instantaneous SERs of the ARelay and B-Relay channel, respectively. $p_{a r}$ and $p_{b r}$ are the probabilities to choose direct DMF. $P_{a b r}$ is the instantaneous SER of the differential DMF and $p_{a b r}$ is the probability to choose such scheme.

For simplicity, (21) is divided into two parts: the differential DMF part and the direct DMF part.

$$
\mathrm{E}\left\{P_{r}\right\}=\mathrm{E}\left\{P_{D i f}\right\}+\mathrm{E}\left\{P_{D i r}\right\},
$$

where

$$
\begin{aligned}
& \mathrm{E}\left\{P_{D i f}\right\}=\int_{\alpha} \int_{\beta} P_{a b r} p_{a b r} f(\alpha) f(\beta) \mathrm{d} \alpha \mathrm{d} \beta, \\
& \mathrm{E}\left\{P_{D i r}\right\}=\int_{\alpha} \int_{\beta}\left(P_{a r} p_{a r}+P_{b r} p_{b r}\right) f(\alpha) f(\beta) \mathrm{d} \alpha \mathrm{d} \beta .
\end{aligned}
$$

The closed form of average symbol error rate can be found in [24] and is neglected in this short paper. Numerical results are provided in the next section.

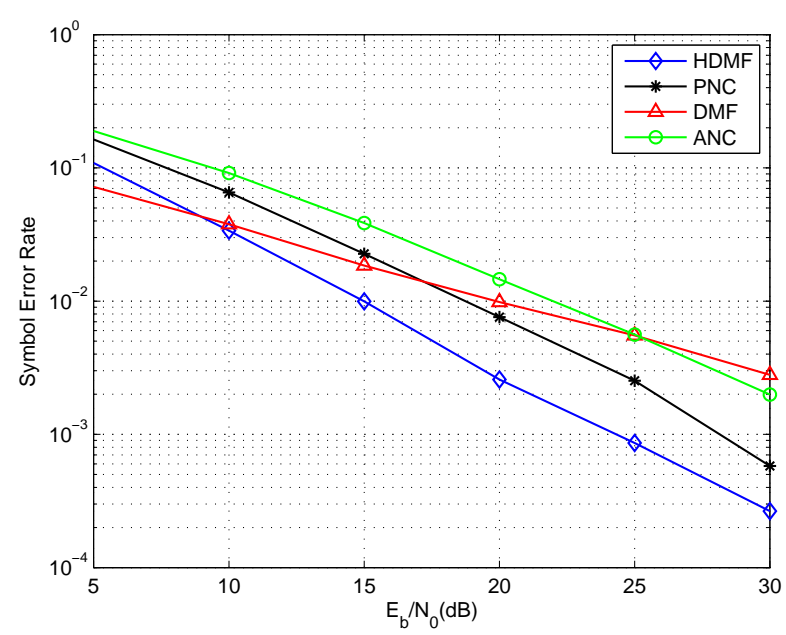

Fig. 2. SER performance of the four protocols.

\section{NUMERICAL EXPERIMENTS}

We investigate the performance of the proposed HDMF protocol through numerical experiments, where the channels are modelled as block Rayleigh distributions and the noise is modelled as additive white Gaussian distributions. The average fading channel gains are fixed to 1 and the SNR $E_{b} / N_{0}$ range is from $5 \mathrm{~dB}$ to $30 \mathrm{~dB}$. We further assume reciprocal channel settings. The modulation is QPSK and each packet has 128 symbols. At every experiment, we generate $10^{5}$ packets that are transmitted from one source node to the other via Relay.

Fig. 2 demonstrates the average SERs of the four protocols. All of them have decreasing SER when SNR increases. However, at the lower SNR region $\left(E_{b} / N_{0}<10 \mathrm{~dB}\right)$, DMF has lower SER than the other protocols. With the increase of SNR, the SERs of HDMF and PNC drop much faster than DMF. After 25dB, even ANC has a lower SER than DMF. For the regions with SNR $>10 \mathrm{~dB}$, HDMF outperforms PNC, ANC and DMF.

Fig. 3 compares the average SER performance between simulation and theoretical analysis (17). From the figure, we can see that simulation result matches well with theoretical analysis, particularly at high SNR regions for the reason that a high SNR assumption is used in the theoretical analysis. Such results confirm the performance of the proposed protocol.

\section{DISCUSSION}

To apply this protocol in $5 \mathrm{G}$ systems requires an efficient scheduling scheme. The queue length at each node, e.g. A, B and $\mathrm{R}$, is an important index of scheduling schemes. Detailed analysis can be carried out by investigating the queue state of each node and the state transition probabilities between two consecutive time slots. Due to the page limit, the analysis is not included in this paper.

The proposed HDMF does not try to solve the fundamental problem of the signal to interference ratio being too low, rather it avoids the problem by relaying the signals through differential DMF rather than detecting the information forcefully which might be degraded by interference and noise. In this 


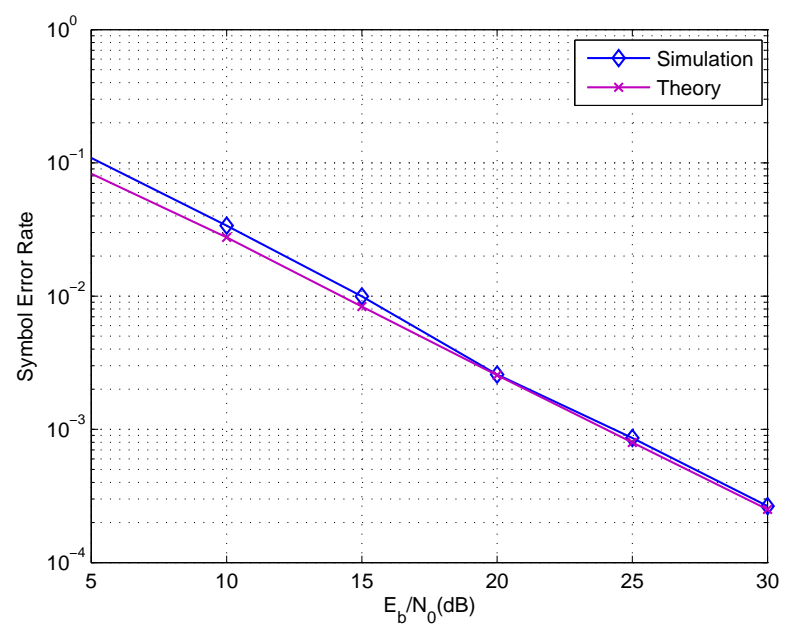

Fig. 3. Average SER comparison.

case, differential DMF provides more information for destination to be able to complete the final symbol detection, e.g. destination can reduce its own contribution in the interference.

If the two channels of TWRC can be synchronized perfectly, the performance of ANC and PNC would have better performance. However, this could be a high requirement since practical wireless channels cannot always guarantee the simultaneous arrival of signals from two distributed source nodes. The proposed HDMF can be switched to direct DMF to avoid the severely degraded channel, thus does not have the limitation of ANC and PNC.

\section{CONCLUSION}

This paper proposes an HDMF protocol for TWRC models which can be applied in future 5G systems for achieving higher data rate. We study the components of HDMF and the fundamentals of direct DMF, differential DMF and the key detection criterion. Comparing its performance with existing protocols shows that the proposed HDMF can achieve a lower average error rate.

\section{ACKNOWLEDGEMENT}

This paper is sponsored by the Research Council UK Digital Economy Theme Sustainable Society Network+ and Royal Society-NSFC Grant No. IE131036.

\section{REFERENCES}

[1] J. Cao, M. Ma, H. Li, Y. Zhang, and Z. Luo, "A survey on security aspects for lte and lte-a networks," IEEE Communications Surveys Tutorials, vol. 16, no. 1, pp. 283-302, First 2014.

[2] Z. Chen, T. Lim, and M. Motani, "Fading two-way relay channels: Physical-layer versus digital network coding," IEEE Trans. Wireless Commun., vol. PP, no. 99, pp. 1-1, 2014.

[3] D. Zeng, S. Guo, Y. Xiang, and H. Jin, "On the throughput of two-way relay networks using network coding," IEEE Trans. Parallel Distrib. Syst., vol. 25, no. 1, pp. 191-199, Jan 2014.

[4] S. Cameron, S. Hailes, S. McClean, and et al., "SUAAVE: Combining aerial robots and wireless networking," in SUAAVE. University of Oxford, University College London, University of Ulster, Feb. 2010, pp. 1-14.
[5] C. Luo, S. McClean, G. Parr, Q. Wang, X. Wang, and C. Grecos, "A communication model to decouple the path planning and connectivity optimization and support cooperative sensing," IEEE Trans. Veh. Technol., vol. 63, no. 8, pp. 3985-3997, Oct 2014.

[6] Z. Chen and H. Liu, "Spectrum-efficient coded modulation design for two-way relay channels," IEEE J. Sel. Areas Commun., vol. 32, no. 2, pp. 251-263, February 2014.

[7] H. Falaki, D. Lymberopoulos, R. Mahajan, S. Kandula, and D. Estrin, "A first look at traffic on smartphones," in Proceedings of the 10th ACM SIGCOMM conference on Internet measurement, ser. IMC '10. New York, NY, USA: ACM, 2010, pp. 281-287.

[8] D. G. Jeong and W. S. Jeon, "Time slot allocation in CDMA/TDD systems for mobile multimedia services," IEEE Commun. Lett., vol. 4, no. 2, pp. $59-61$, Feb. 2000.

[9] C. E. Shannon, "Two-way communication channels," Proc. 4th Berkeley Symp. Math. Stat. and Prob., vol. 1, pp. 611-644, 1961.

[10] T. M. Cover and A. A. E. Gamal, "Capacity theorems for the relay channel," IEEE Trans. Inf. Theory, vol. IT-25, pp. 572-584, Sept. 1979.

[11] A. Sendonaris, E. Erkip, and B. Aazhang, "User cooperation diversitypart i: System description," IEEE Trans. Commun., vol. 51, pp. 19271938, Nov. 2003

[12] J. N. Laneman, D. N. C. Tse, and G. W. Wornell, "Cooperative diversity in wireless networks: Efficient protocols and outage behavior," IEEE Trans. Inf. Theory, vol. 50, no. 12, pp. 3062-3080, Dec. 2004.

[13] T. Wang, A. Cano, G. Giannakis, and J. Laneman, "High-performance cooperative demodulation with decode-and-forward relays," IEEE Trans. Commun., vol. 55, no. 7, pp. 1427-1438, July 2007.

[14] S. Katti, S. Gollakota, and D. Katabi, "Embracing wireless interference: Analog network coding," in in ACM SIGCOMM. MIT, 2007, pp. 397408.

[15] S. Zhang, S. C. Liew, and P. P. Lam, "Hot topic: physical-layer network coding," in MobiCom '06: Proceedings of the 12th annual international conference on Mobile computing and networking. New York, NY, USA: ACM Press, 2006, pp. 358-365.

[16] Z. Chen, T. J. Lim, and M. Motani, "Digital network coding aided twoway relaying: Energy minimization and queue analysis," IEEE Trans. Wireless. Commun., vol. 12, no. 4, pp. 1947-1957, 2013.

[17] Y. Gong, C. Luo, and Z. Chen, "Two-path succussive relaying with hybrid demodulate and forward," IEEE Trans. Veh. Technol., vol. 61, no. 5, pp. $2044-2053$, jun 2012.

[18] M. Noori and M. Ardakani, "On symbol mapping for binary physicallayer network coding with PSK modulation," IEEE Trans. Wireless Commun., vol. 11, no. 1, pp. 21-26, January 2012.

[19] A. Li, Y. Yan, and H. Kayama, "An enhanced denoise-and-forward relaying scheme for fading channel with low computational complexity," IEEE Signal Process. Lett., vol. 15, pp. 857-860, 2008.

[20] T. Koike-Akino, P. Popovski, and V. Tarokh, "Optimized constellations for two-way wireless relaying with physical network coding," IEEE J. Sel. Areas Commun., vol. 27, no. 5, pp. 773-787, June 2009.

[21] H. J. Yang, Y. Choi, and J. Chun, "Modified high-order PAMs for binary coded physical-layer network coding," IEEE Commun. lett., vol. 14 no. 8, pp. 689-691, August 2010.

[22] S. Lin, J. Costello, D.J., and M. Miller, "Automatic-repeat-request errorcontrol schemes," IEEE Commun. Mag., vol. 22, no. 12, pp. 5-17, December 1984

[23] B. Sklar, Digital communications: fundamentals and applications, ser. Prentice Hall Communications Engineering and Emerging Technologies Series. Prentice-Hall PTR, 2001.

[24] C. Luo, C. Peoples, G. Parr, S. McClean, and X. Wang, "Hybrid demodulate-forward relay protocol for two-way relay channels," IEEE Trans. Wireless Commun. (In press), 2015. [Online]. Available: http://arxiv.org/submit/1219649 